\title{
Endoscopic Approaches to the Treatment of Variceal Hemorrhage in Hemodialysis-Dependent Patients
}

\author{
Xiaoquan Huang, ${ }^{1,2}$ Lili Ma, ${ }^{1}$ Xiaoqing Zeng, ${ }^{2}$ Jian Wang, ${ }^{2}$ Jie Chen, ${ }^{2}$ and Shiyao Chen ${ }^{1,2}$ \\ ${ }^{1}$ Endoscopy Center and Endoscopy Research Institute, Zhongshan Hospital, Fudan University, Shanghai 200032, China \\ ${ }^{2}$ Department of Gastroenterology, Zhongshan Hospital, Fudan University, Shanghai 200032, China \\ Correspondence should be addressed to Shiyao Chen; chen.shiyao@zs-hospital.sh.cn
}

Received 26 August 2016; Revised 3 November 2016; Accepted 4 December 2016

Academic Editor: Atsushi Irisawa

Copyright (c) 2016 Xiaoquan Huang et al. This is an open access article distributed under the Creative Commons Attribution License, which permits unrestricted use, distribution, and reproduction in any medium, provided the original work is properly cited.

Background. Esophagogastric variceal hemorrhage leads to challenging situation in chronic kidney disease patients on maintenance hemodialysis. Aims. To determine the safety and efficacy of endoscopic approaches to patients with hemodialysis-dependent concomitant with esophagogastric varices. Methods. Medical records were reviewed from January 1, 2004, to December 31, 2015, in our hospital. Five consecutive hemodialysis-dependent patients with variceal hemorrhage who underwent endoscopic treatments were retrospectively studied. Results. The median age of the patients was 54 years (range 34-67 years) and the median follow-up period was 21.3 months (range 7-134 months). All the patients received a total of three times heparin-free hemodialysis 24 hours before and no more than 24 hours and 72 hours after endoscopic treatment. They successfully had endoscopic variceal ligation, endoscopic injection sclerotherapy, and/or N-butyl cyanoacrylate injection. The short-term efficacy is satisfying and long-term follow-up showed episodes of rebleeding. Conclusions. Endoscopic approaches are the alternative options in the treatment of upper gastroenterology variceal hemorrhage in hemodialysis-dependent patients without severe complications.

\section{Introduction}

The global prevalence and incidence of end-stage renal disease (ESRD) are increasing worldwide while the mortality among patients with ESRD observed large net reductions due to both dialysis and transplantation [1]. Cirrhotic patients with ESRD have increased complications and higher mortality rate [2]. The prevalence of cirrhosis was about $6.2 \%$ at the beginning of dialysis in a cohort study in Taiwan [3]. Esophagogastric variceal bleeding accounts for a mortality rate of approximately $15 \%$ in general population [4]. Evidence had proven endoscopic approaches are the most effective intervention for primary and secondary prevention of gastroesophageal variceal bleeding, recommended as the firstline option [5].

However, patients with marked impaired renal function undergoing hemodialysis and concomitant cirrhotic gastroesophageal varices remained intractable. Their fragile hemostasis states, caused by renal anemia, uremic platelet dysfunction, and use of anticoagulants [6,7], lead to recurrent upper gastroenterology bleeding. Haskal and Radhakrishnan [8] had reported that transjugular intrahepatic portosystemic shunts (TIPS) were effective in controlling ascites and bleeding in dialysis-dependent patients but a high incidence of post-TIPS hepatic encephalopathy. Simultaneous liverkidney transplantation (SLKT) is the best choice for them considering both survival and quality-adjusted life years $[9,10]$. However, it takes time waiting for the donor and some patients died of massive variceal hemorrhage during the waiting period. Endoscopic treatment might be the alternative option to avoid massive variceal bleeding. The concerns of the endoscopic approach are the risk of bleeding and anesthesia management. To our knowledge, no literature reporting endoscopic treatment of varices in hemodialysisdependent patients was found. In this study, we report our experiences in controlling esophagogastric variceal bleeding in hemodialysis-dependent patients. 


\section{Materials and Methods}

2.1. Study Design. In this tertiary hospital-based retrospective case series study, a total of 2038 consecutive hospitalized patients with esophageal and/or gastric varices undergoing endoscopic therapy were screened. We included (1) patients who required maintenance hemodialysis; (2) patients confirmed to have liver cirrhosis by computed tomography; (3) patients confirmed to have varices by gastroscopy; and (4) patients who had endoscopic treatment for secondary prevention of variceal bleeding. We excluded patients who had endoscopic treatment before starting hemodialysis. We retrieved medical records including the emergency room and outpatient department from our hospital between January 1, 2004, and December 31, 2015. The end point was set at March 31, 2016. All the patients were followed up via phone calls and outpatient clinic visits. This retrospective report was approved by the institution's Ethics Committee and written informed consent was obtained from each patient. Median and range were shown to describe quantitative data.

2.2. Endoscopic Intervention. The devices and drugs used included the electronic endoscope GIF-XQ240/260 (Olympus, Tokyo, Japan), 6 multiband ligators (Cook Endoscopy, Winston-Salem, North Carolina, USA) or 7 multiband ligators (Boston Scientific, Natick, Massachusetts, USA), Nbutyl-cyanoacrylate (Beijing Suncon Medical Adhesive, Beijing, China), lauromacrogol (Tianyu Pharmaceutical, China), lipiodol, and injection needle (Olympus NM-200 L-423, Tokyo, Japan).

Gastric varices were treated with N-butyl-cyanoacrylate using the sandwich method (lipiodol or $20 \%$ glucose or lauromacrogol-cyanoacrylate-lipiodol or $20 \%$ glucose or lauromacrogol). Each cyanoacrylate injection point was no more than $2.0 \mathrm{~mL}$ and an equal volume of lipiodol or $20 \%$ glucose or 2-8 $\mathrm{mL}$ lauromacrogol determined by the varix size according to our published study [11]. Multiple sites injection was an attempt to completely obturate the gastric varices in one session. To decrease the risk of a variceal tear, the needle sheath was held in the puncture site to prevent leakage of the cyanoacrylate and to ensure the varice has hardened before retracting the injector catheter.

Endoscopic variceal ligation (EVL) is the primary treatment selection for esophageal varices according to guideline and our experiences $[12,13]$. Ligation was applied from $1 \mathrm{~cm}$ above the Z-line in a spirally ascending fashion, with no more than six or seven bands used per session. Endoscopic injection sclerosis (EIS) treatment is usually performed in patients who had multiple EVL sessions and as an attempt to eliminate the small esophageal varix. The initial injection started above the Z-line, and intravariceal or paravariceal injection of $10-30 \mathrm{~mL}$ per session of lauromacrogol was injected. Follow-up endoscopy was performed at an interval of no less than 2 months and treatment was repeated until complete obliteration was achieved.

2.3. Perioperative Management. All the patients required hemodialysis three times per week. Heparin-free dialysis was performed the day before the endoscopic procedure and postoperative heparin-free dialysis was performed no more than 24 hours after the procedures, and the third heparinfree dialysis was done more than 72 hours after the procedure. Patients did not receive any other anticoagulation drugs for a week. Later comes the routine usage of heparin dialysis. They were adequately treated with proton pump inhibitors (PPIs) after endoscopic treatment and no prophylactic antibiotics were used.

2.4. Anesthesia Management. The patient's general condition should be designated as American Society of Anesthesiologists (ASA) classification I, II, or III, and these patients underwent propofol sedation for endoscopy [14]. The initial dosage was $2 \mathrm{mg} / \mathrm{kg}$, and $1 \mathrm{mg} / \mathrm{kg}$ was added by the anesthesiologist if body movement disturbed the procedure. Electrocardiogram, oxygen saturation, respiratory rate, and noninvasive blood pressure measurements were monitored during the endoscopic treatment.

2.5. Statistical Analysis. Statistical analysis was performed with SPSS 23.0 software (SPSS Inc., Chicago, Illinois, USA). Median and range were shown to describe continuous data.

\section{Results}

Five patients were enrolled in this study, and their demographic data were shown in Table 1. Of the five patients, there were three males and two females with the age of 54 years (range, 33-67 years) and the creatinine of $738 \mu \mathrm{mol} / \mathrm{L}$ (range, $542-1131 \mu \mathrm{mol} / \mathrm{L}$ ) at the time of having the first endoscopic treatment. The etiologies of liver cirrhosis included hepatic $\mathrm{B}$ virus (HBV), hepatic $\mathrm{C}$ virus ( $\mathrm{HCV})$, and drug-inducedliver-injury (DILI) (aristolochic acid and unknown herb in traditional Chinese medicine). The primary diseases related to renal failure are chronic glomerulonephritis (80\%) and aristolochic acid nephropathy (20\%). Among these patients, one had hepatocellular carcinoma (HCC, patient 1) and another one had colorectal cancer (CRC, patient 4) before developing into gastroesophageal varices and starting maintenance hemodialysis. The liver function of these patients was mild impairment.

Among the five cases, the time interval between hemodialysis and first endoscopic treatment was 37.5 months (range 5.9-94.6 months). A total of 16 endoscopic procedures were performed including 11 EVLs, 2 EISs, and 3 $\mathrm{N}$-butyl cyanoacrylate injections. The details of endoscopic treatment were shown in Table 2. All the procedures were secondary prophylaxis of bleeding. The changes before and after endoscopic treatment of gastric varices were shown in Figure 1. All the three cyanoacrylate injection procedures had immediate blood exudations and were controlled by rinsing with $8 \%$ ice norepinephrine solution. The esophageal varices were treated by ligations and sclerotherapy (Figures 2(a), 2(b), and 2(c)). No delay bleeding was observed when they were back to the ward. No complications such as infections, massive bleeding, or stroke were observed in these patients. 


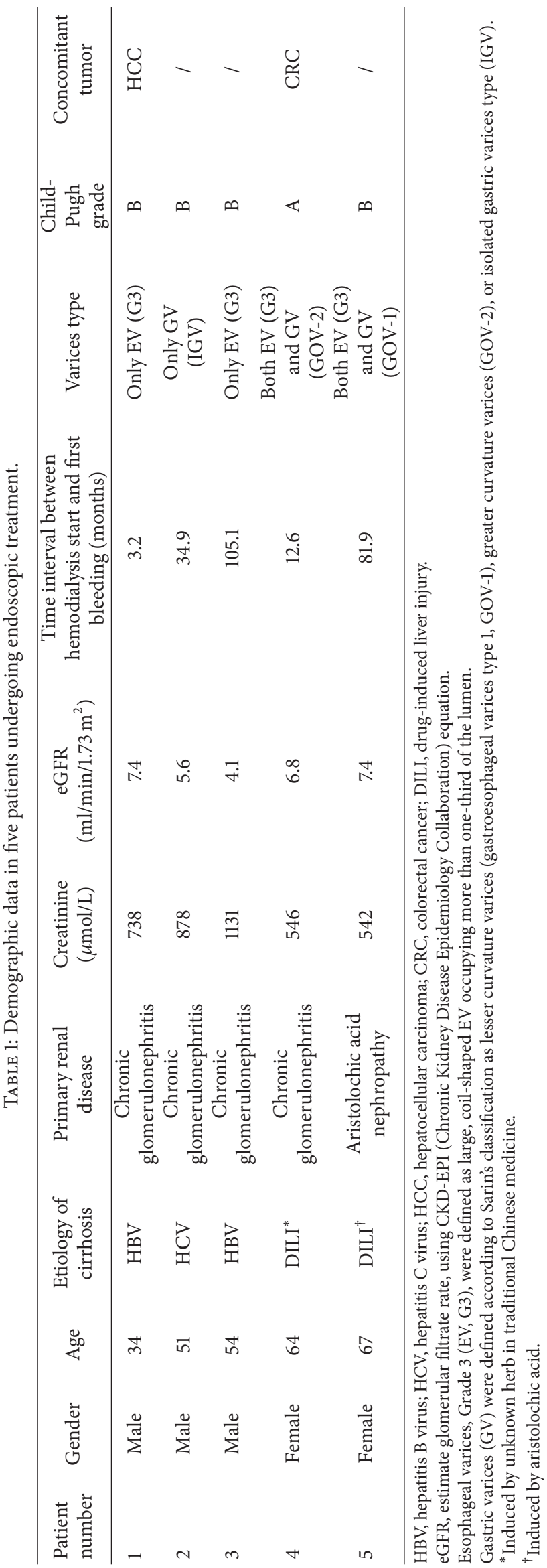




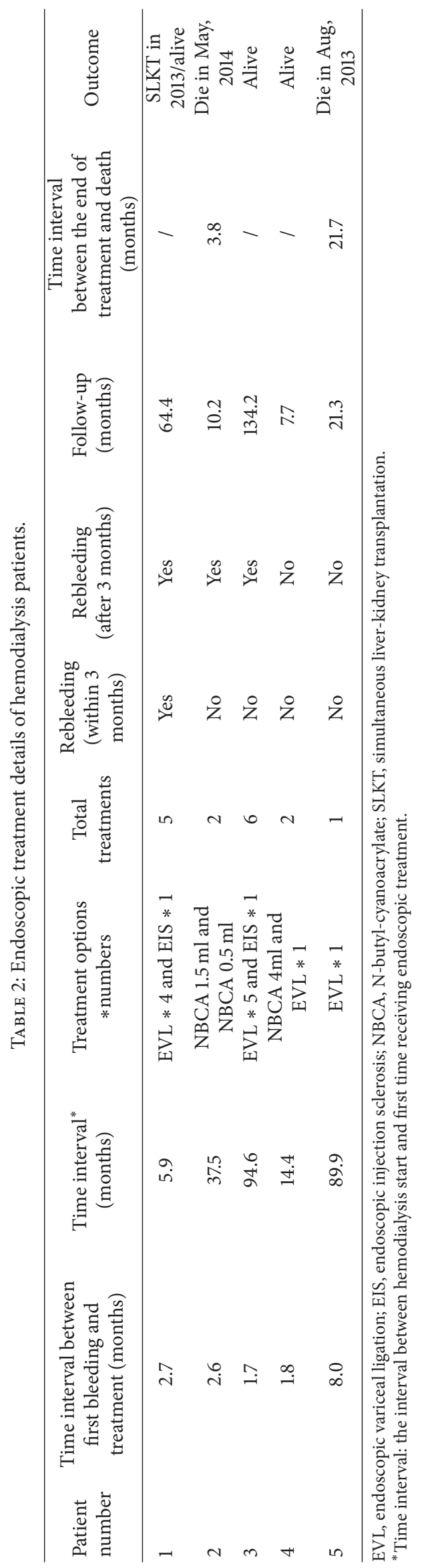




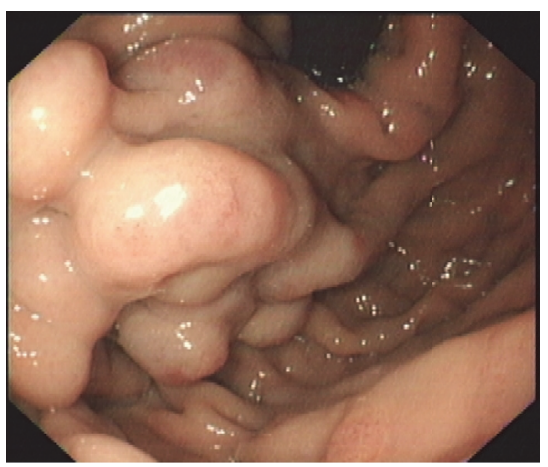

(a)

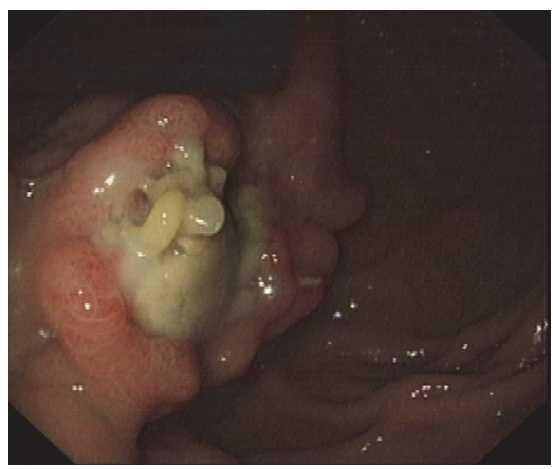

(d)

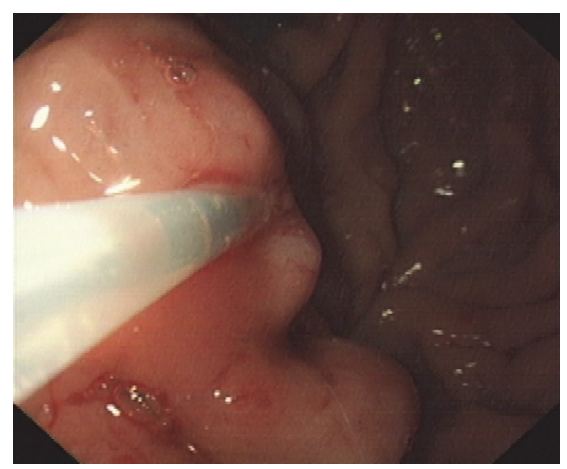

(b)

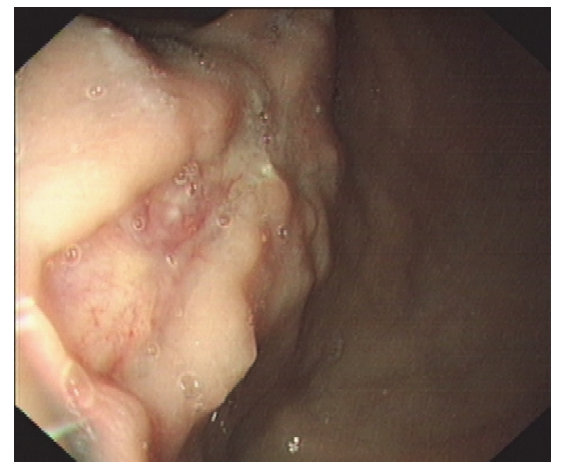

(e)

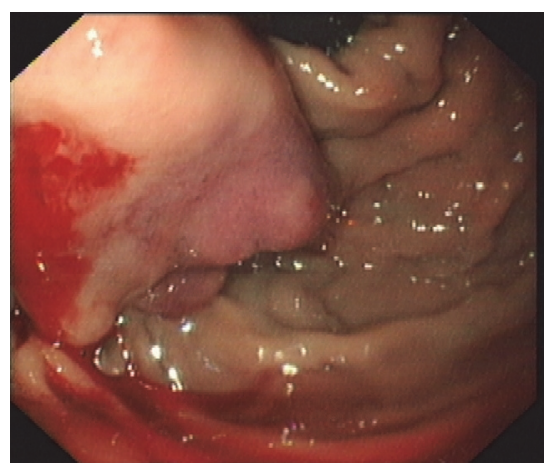

(c)

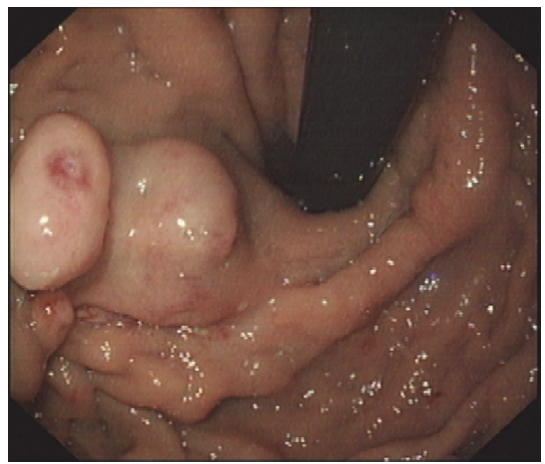

(f)

FIGURE 1: Gastric varices. (a) Isolate gastric varices vein $(4 \mathrm{~cm})$ before treatment. (b) Injecting $6 \mathrm{~mL}$ lauromacrogol $+1.5 \mathrm{~mL}$ N-butylcyanoacrylate $+4 \mathrm{~mL}$ lauromacrogol. (c) Mild blood exudation after injection; the injection site was rinsed with ice-cold norepinephrine. (d) Gastric ulceration in the injection site. (e) Scar of cyanoacrylate injection. (f) Recurrence of gastric varices in 10 months (patient 2).

Of the five patients, only one (20\%) experienced rebleeding within 3 months after the endoscopic treatment and three $(60 \%)$ suffered from nonlethal rebleeding episodes after 3 months. The median follow-up period was 21.3 months (range 7-134 months). Patient 1 received SLKT in 2013 and completely cured without severe complications (Figure 2(d)).

\section{Discussion}

An increasing number of patients with concurrent ESRD and varices make the management of variceal hemorrhage intractable. The concerns of endoscopic approaches are the risk of bleeding and anesthesia. Our experiences illustrate that endoscopic treatment is effective and relatively safe in these patients. All of our patients' esophageal varices were prominent in the distal esophagus, in contrast to the "downhill varices" in the proximal esophagus in hemodialysis patient with central venous catheters [15]. "Uphill" varices caused by cirrhotic portal hypertension are much easy to bleed. Endoscopic approaches are promising in the hemorrhage management [16].

While the impaired renal and hepatic functions increased the risk of sedation and propofol, an ultrashort acting hypnotic agent was given. Propofol is widely recommended in short endoscopic procedures and superior in terms of patient's tolerance, maximum level of sedation achieved, and shorter recovery room times [17]. Only very little part of propofol clearance is through renal elimination. Its pharmacokinetics are minimally changed in patients with impaired liver and renal functions [18]. Patients sedated with propofol were confirmed to be safe and less invasive by a large amount of patients undergoing endoscopic treatments in our hospital. Closely monitoring the blood pressure and limitation of fluid infusion are required.

Because none of our patients had endoscopic procedures for the management of acute variceal hemorrhage, propofol sedation is possible in secondary prophylaxis for variceal hemorrhage. The sedation helps with the compliable of patients during treatments and reduces the possibility of bleeding and makes bleeding easy to control during injection. Extravascular injection of drugs is easy to cause gastric ulceration and precise intravascular injection can also reduce rebleeding risk [19]. In addition, the administration of propofol was by anesthesiologists, and we can immediately switch into intubation when necessary.

Little is known about the safety of long-term heparin-free hemodialysis; however, temporarily heparin-free hemodialysis without anticoagulation drug is beneficial to these patients for endoscopic procedures. Perioperative heparin-free dialysis reduced the risk of bleeding during endoscopic treatment and delay bleeding. 


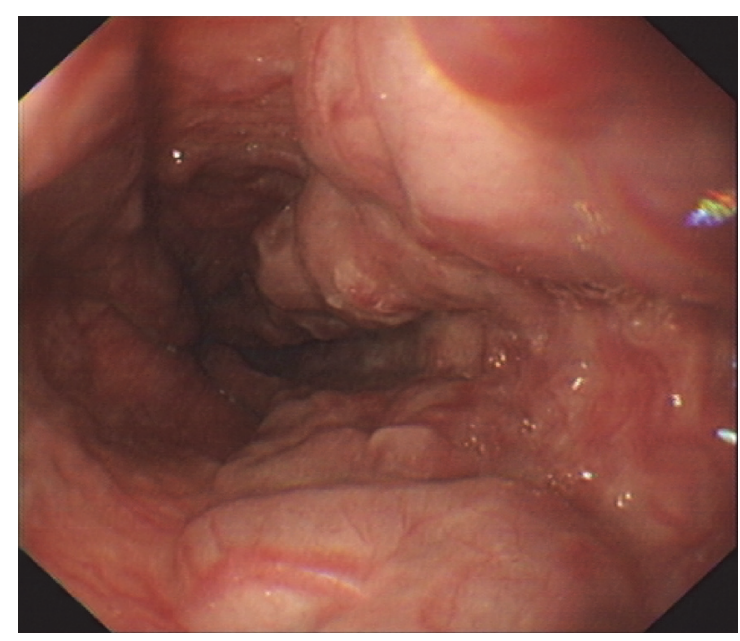

(a)

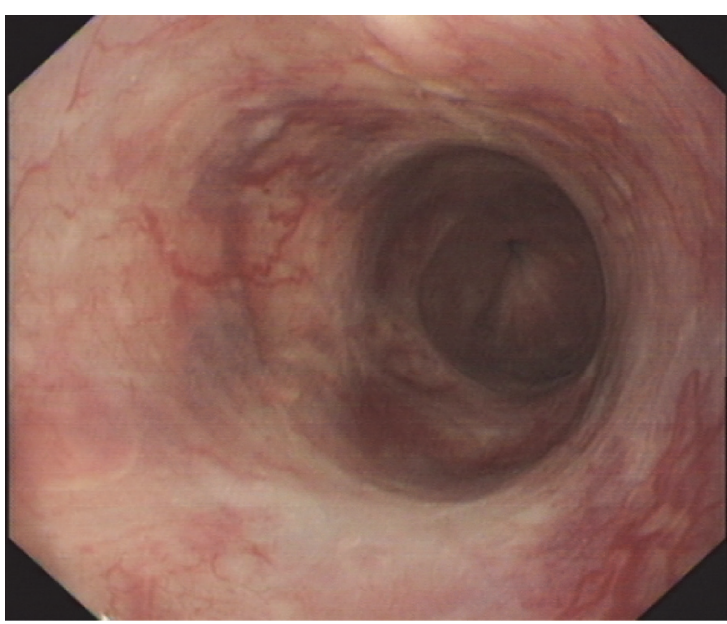

(c)

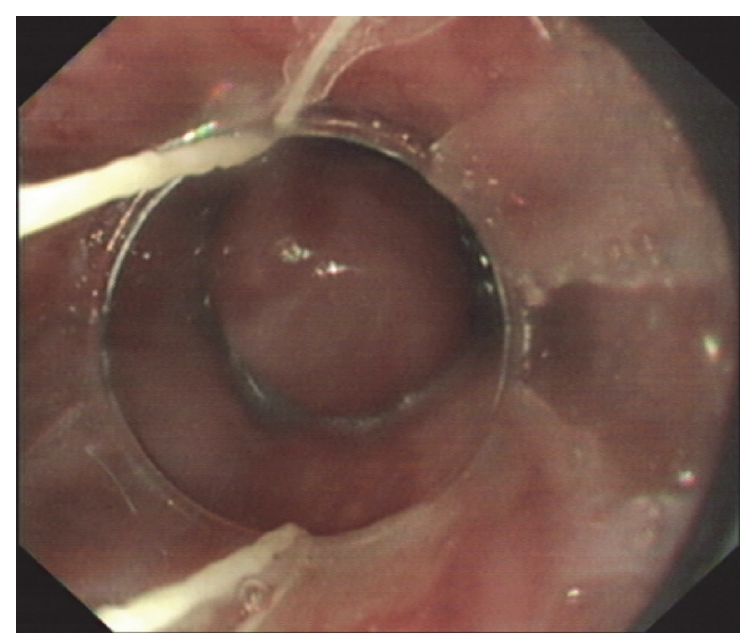

(b)

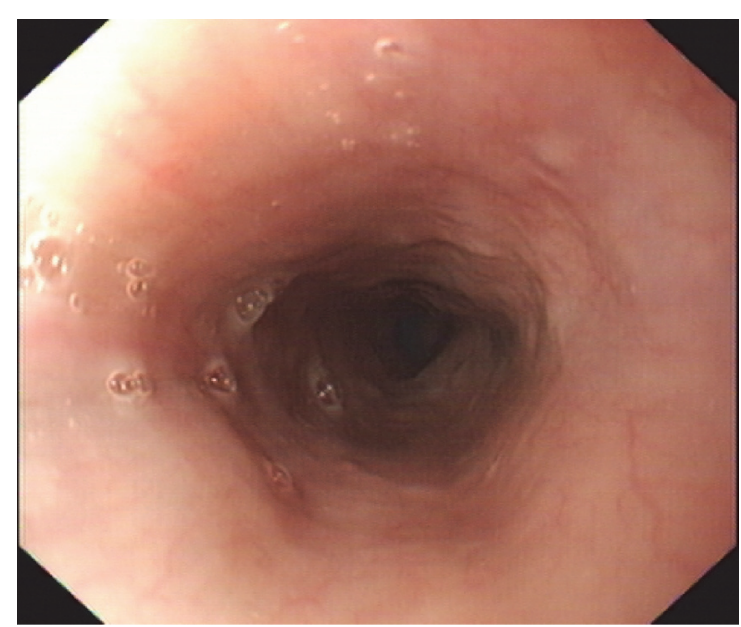

(d)

FIGURE 2: Esophageal varices. (a) Esophageal appearance before treatment. (b) Endoscopic variceal ligations. (c) Esophageal appearance after endoscopic variceal ligation procedures. (d) Brand new esophageal appearance after simultaneous liver-kidney transplantation (patient 1).

Long-term rebleeding episodes might be because of the high portal pressure in these patients. However, because of their poor general conditions, none of them had hepatic venous pressure gradient (HVPG) measurement. The actual pressure of these patients remained unknown. Further study will be required to evaluate the long-term outcomes in more patients.

In conclusion, for hemodialysis-dependent patients with esophagogastric varices, endoscopic approaches might be the alternative options to TIPS and SKLT, which reduce the risk of variceal hemorrhage without severe complications.

\section{Disclosure}

The funder had no role in the study design, data collection and analysis, decision to publish, or preparation of the manuscript.

\section{Competing Interests}

The authors declare that they have no competing interests.

\section{Acknowledgments}

The authors would like to thank all the staff in Department of Gastroenterology, Endoscopy Center and Endoscopy Research Institute, Department of Anesthesiology, and Department of Nephropathy of Zhongshan Hospital, Fudan University, for assistance. The study was supported by Innovation Fund of Shanghai Committee of Science and Technology (no. 15411950501).

\section{References}

[1] R. Saran, Y. Li, B. Robinson et al., "US renal data system 2015 annual data report: epidemiology of kidney disease in the 
United States," American Journal of Kidney Diseases, vol. 67, no. 3, supplement 1, pp. A7-A8, 2016.

[2] T.-H. Hung, C.-C. Tsai, K.-C. Tseng et al., "High mortality of cirrhotic patients with end-stage renal disease," Medicine, vol. 95, no. 10, Article ID e3057, 2016.

[3] C.-C. Chien, J.-J. Wang, Y.-M. Sun et al., "Long-term survival and predictors for mortality among dialysis patients in an endemic area for chronic liver disease: A National Cohort Study in Taiwan," BMC Nephrology, vol. 13, no. 1, article 43, 2012.

[4] M. Bai, X. Qi, M. Yang, G. Han, and D. Fan, "Combined therapies versus monotherapies for the first variceal bleeding in patients with high-risk varices: a meta-analysis of randomized controlled trials," Journal of Gastroenterology and Hepatology, vol. 29, no. 3, pp. 442-452, 2014.

[5] R. de Franchis and B. V. Faculty, "Expanding consensus in portal hypertension: report of the Baveno VI Consensus Workshop: stratifying risk and individualizing care for portal hypertension," Journal of Hepatology, vol. 63, no. 3, pp. 743-752, 2015.

[6] P. Sood, G. Kumar, R. Nanchal et al., "Chronic kidney disease and end-stage renal disease predict higher risk of mortality in patients with primary upper gastrointestinal bleeding," American Journal of Nephrology, vol. 35, no. 3, pp. 216-224, 2012.

[7] G. Escolar, M. Díaz-Ricart, and A. Cases, "Uremic platelet dysfunction: past and present," Current Hematology Reports, vol. 4, no. 5, pp. 359-367, 2005.

[8] Z. J. Haskal and J. Radhakrishnan, “Transjugular intrahepatic portosystemic shunts in hemodialysis-dependent patients and patients with advanced renal insufficiency: safety, caution, and encephalopathy," Journal of Vascular and Interventional Radiology, vol. 19, no. 4, pp. 516-520, 2008.

[9] M. M. Doyle, V. Subramanian, N. Vachharajani et al., "Results of simultaneous liver and kidney transplantation: a single-center review," Journal of the American College of Surgeons, vol. 223, no. 1, pp. 193-201, 2016.

[10] B. Kiberd, C. Skedgel, I. Alwayn, and K. Peltekian, "Simultaneous liver kidney transplantation: a medical decision analysis," Transplantation, vol. 91, no. 1, pp. 121-127, 2011.

[11] X. Zeng, L. Ma, Y. Tzeng et al., "Endoscopic cyanoacrylate injection with or without lauromacrogol for gastric varices: a randomized pilot study," Journal of Gastroenterology and Hepatology, 2016.

[12] J. Chen, X.-Q. Zeng, L.-L. Ma et al., "Randomized controlled trial comparing endoscopic ligation with or without sclerotherapy for secondary prophylaxis of variceal bleeding," European Journal of Gastroenterology \& Hepatology, vol. 28, no. 1, pp. 95100, 2016.

[13] J. Chen, X. Q. Zeng, L. L. Ma et al., "Long-term efficacy of endoscopic ligation plus cyanoacrylate injection with or without sclerotherapy for variceal bleeding," Journal of Digestive Diseases, vol. 17, no. 4, pp. 252-259, 2016.

[14] B. K. Enestvedt, G. M. Eisen, J. Holub, and D. A. Lieberman, "Is the American Society of Anesthesiologists classification useful in risk stratification for endoscopic procedures?" Gastrointestinal Endoscopy, vol. 77, no. 3, pp. 464-471, 2013.

[15] L. Gessel and J. Alcorn, "Variants of varices: is it all 'downhill' from here?” Digestive Diseases and Sciences, vol. 60, no. 2, pp. 316-319, 2015.

[16] C. Froilán, L. Adán, J. M. Suárez et al., “Therapeutic approach to "downhill" varices bleeding," Gastrointestinal Endoscopy, vol. 68, no. 5, pp. 1010-1012, 2008.
[17] D. O. Faigel, T. H. Baron, J. L. Goldstein et al., "Guidelines for the use of deep sedation and anesthesia for GI endoscopy," Gastrointestinal Endoscopy, vol. 56, no. 5, pp. 613-617, 2002.

[18] J. Kanto and E. Gepts, "Pharmacokinetic implications for the clinical use of propofol," Clinical Pharmacokinetics, vol. 17, no. 5, pp. 308-326, 1989.

[19] L. Cheng, Z. Wang, C. Li, W. Lin, A. E. Yeo, and B. Jin, "Low incidence of complications from endoscopic gastric variceal obturation with butyl cyanoacrylate," Clinical Gastroenterology and Hepatology, vol. 8, no. 9, pp. 760-766, 2010. 


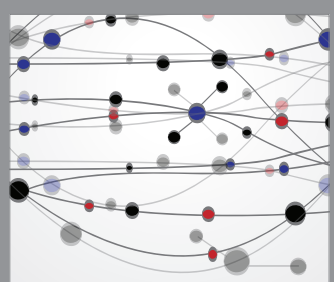

The Scientific World Journal
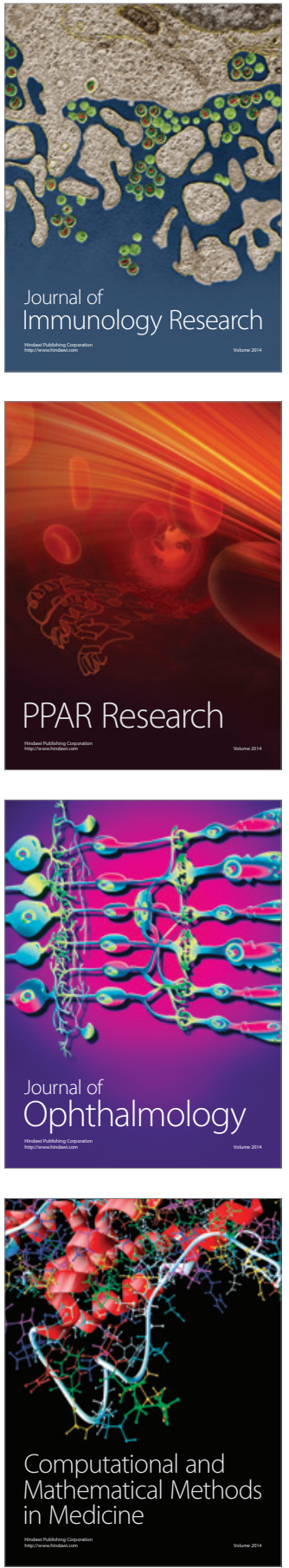

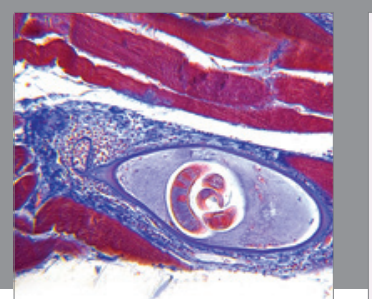

Gastroenterology Research and Practice

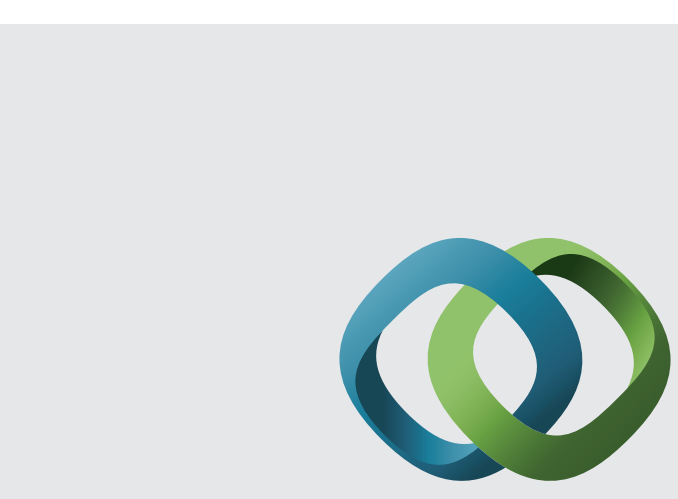

\section{Hindawi}

Submit your manuscripts at

http://www.hindawi.com
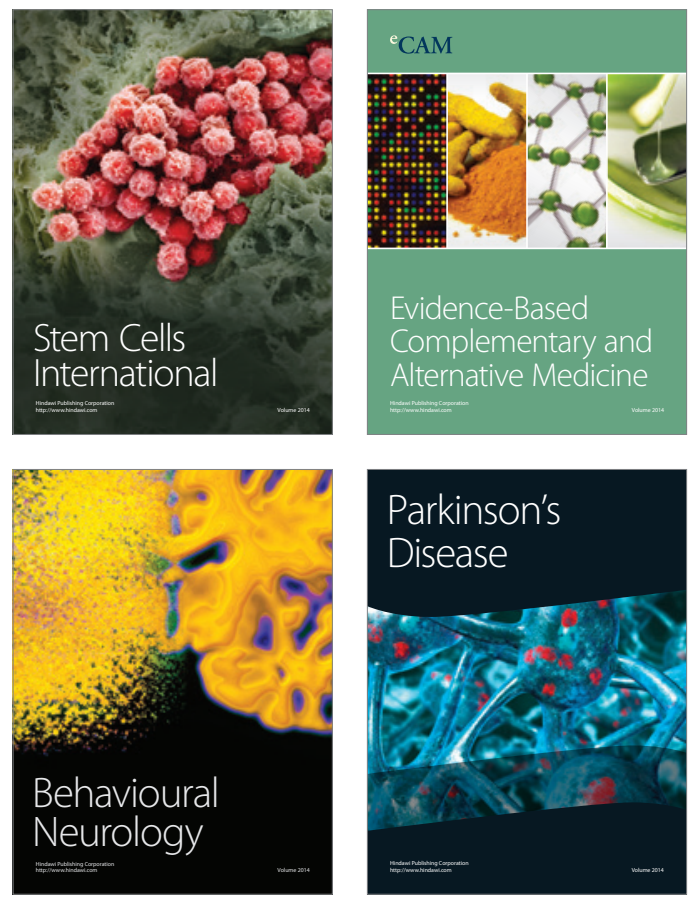
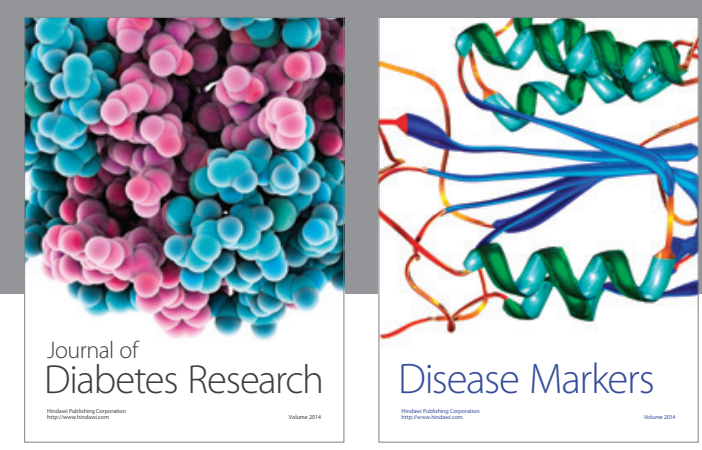

Disease Markers
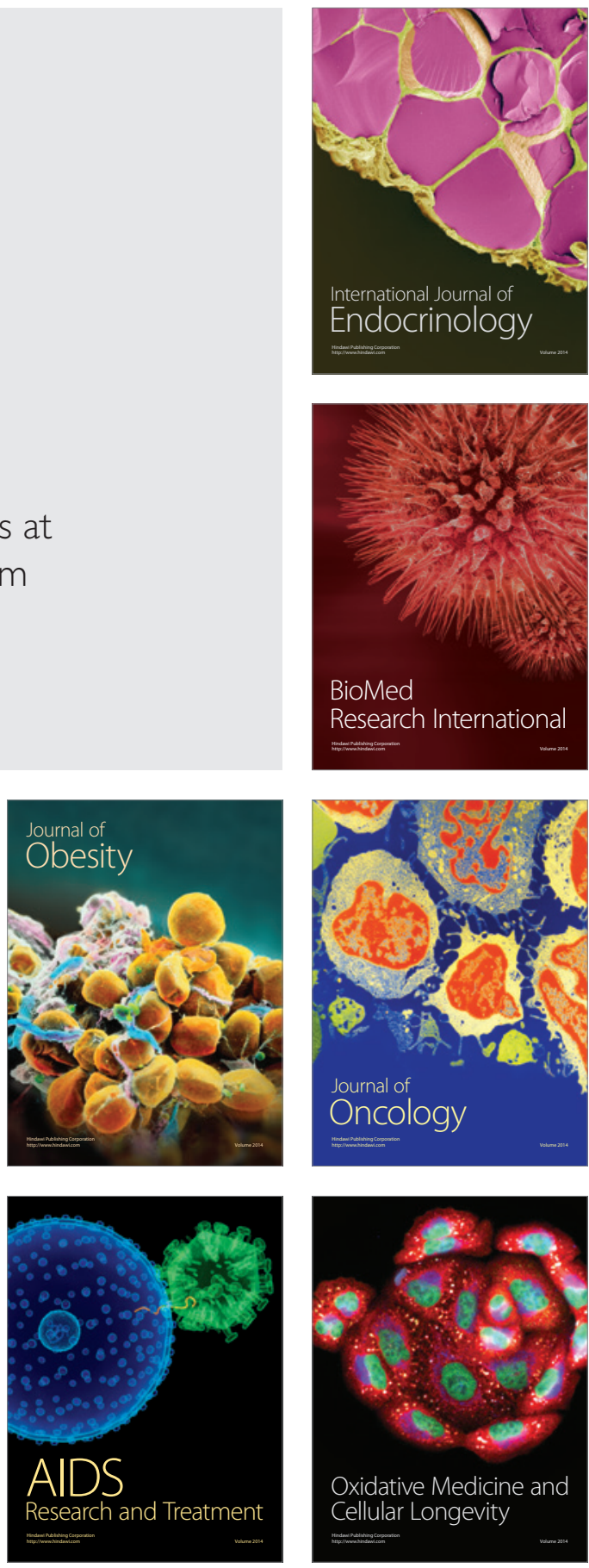